\title{
Promoción y prevención del cáncer de cérvix en Huancayo
}

\section{Campaign for cenvical cancer prevention in Huancayo}

\author{
Arleth Mejia Cuyatti \\ Universidad Continental
}

\author{
Leticia Ribbeck Soto ${ }^{2}$ \\ Universidad Continental \\ leth.ribbeck95@gmail.com
}

\author{
Nataly Romero Manrique ${ }^{3}$ \\ Universidad Continental
}

\section{RESUMEN}

El objetivo ha sido sensibilizar a la población del barrio de Incho en el distrito de El Tambo en Huancayo sobre la prevención del cáncer de cérvix, asimismo difundir a la población sobre la importancia del tamizaje de Papanicolaou. En las actividades previas se utilizó la espina de Ishikawa y el cuadro de priorización de problemas para identificar las causas por las cuales la población femenina expuesta al cáncer de Cérvix no conoce cómo prevenir esta enfermedad. Para cumplir el objetivo se realizó campañas de capacitación a la población a través de material impreso con información completa de prevención de cáncer de Cérvix, también se aplicó un cuestionario para recoger datos respecto al conocimiento de la enfermedad. Como resultado se encontró que la mayoría de las mujeres no habían recibido este tipo de charlas, más del $50 \%$ no conocía maneras de prevenir el cáncer de Cérvix, sin embargo la mayoría tenía conocimiento del Test de Papanicolau; asimismo la mayor parte de las entrevistadas después de la sensibilización se sentían mas satisfechas respecto a las dudas que tenían sobre el cáncer de Cérvix. En conclusión estos programas de sensibilización a la población ayudan a poblaciones vulnerables a que puedan prevenir esta enfermedad, y contribuyen a mejorar el compromiso de asistencia de las mujeres a los centros de salud para realizar sus pruebas respectivas.

Palabras clave: Cáncer de Cérvix, test de Papanicolau, factores de riesgo.

\begin{abstract}
The study goal was raising awareness in the Incho neighborhood, El Tambo district from Huancayo about cervical cancer prevention, and also spread this awareness to the population on the importance of Papanicolau smear screening. In Previous activities, Ishikawa spine and the prioritizing problems table were used to identify the reasons why female population exposed to cervical cancer does not know how to prevent this disease. In order to reach the goal, advocacy campaign efforts were performed to meet the population target through printed material with full information on cervical cancer prevention; a questionnaire to collect data regarding the disease knowledge was also used. As a Result, most women had not received this kind of information, more than $50 \%$ did not know how to prevent cervical cancer, however, most of them knew about the Papanicolau Test. Likewise, after sensitization the majority of the examined women had their doubts satisfied regarding cervical cancer. Finally, these sensitization programs help prevent the spread of disease on vulnerable populations, and improve women's agreement to attend health centers for their respective check-ups.
\end{abstract}

Keywords: Cervical cancer, pap test, risk factors.

Historial del artículo:

Recibido: 21 de octubre de 2015. Aprobado: 20 de noviembre de 2015. Disponible en línea: 30 de diciembre de 2015

1 Estudiante de Medicina Humana de la Universidad Continental.

2 Estudiante de Medicina Humana de la Universidad Continental.

3 Estudiante de Medicina Humana de la Universidad Continental. 


\section{INTRODUCCIÓN}

A nivel mundial, el cáncer cervical es el tercer tipo de cáncer más común en las mujeres. Es mucho menos común en los Estados Unidos debido al uso rutinario de citologías vaginales (pruebas de Papanicolau). Los cánceres cervicales comienzan en las células de la superficie del cuello uterino.

Existen dos tipos de células en dicha superficie: escamosas y columnares. La mayoría de los cánceres de cuello uterino provienen de las células escamosas.

El desarrollo del cáncer cervical generalmente es muy pueden causar verrugas genitales, mientras que otras no causan ningún problema en absoluto. Los hábitos y patrones sexuales de una mujer pueden aumentar su riesgo de padecer cáncer cervical.

Las prácticas sexuales riesgosas abarcan:

- Tener relaciones sexuales a una edad temprana.

- Tener múltiples parejas sexuales.

- Tener una pareja o múltiples parejas que participen en actividades sexuales de alto riesgo.

El cáncer cervical se puede prevenir haciendo lo siguiente:



Figura $\mathrm{N}^{\circ} 1$ : Mortalidad por cáncer de cuello uterino en países de América Latina.

Fuente: http://sisbib.unmsm.edu.pe/bvrevistas/ginecologia/vol53_n2/pdf/A05V53N2.pdf

lento y comienza como una afección precancerosa llamada displasia. Esta afección se puede detectar por medio de una citología vaginal y es $100 \%$ curable. Pueden pasar años para que los cambios se conviertan en cáncer cervical. La mayoría de las mujeres a quienes se les diagnostica cáncer cervical en la actualidad no se han sometido a citologías vaginales regulares o no han tenido un seguimiento por resultados anormales en éstas.

Casi todos los cánceres cervicales son causados por el virus del papiloma humano (VPH), un virus común que se disemina a través de las relaciones sexuales. Existen muchos tipos diferentes (cepas) de VPH y algunas cepas llevan a cáncer cervical. Otras cepas
- Hacerse aplicar la vacuna contra el VPH. Previene la mayoría de los tipos de infecciones por el VPH que causan cáncer de cuello uterino. El médico puede decirle si la vacuna es apropiada en su caso.

- Practicar relaciones sexuales con protección. El uso del condón durante la relación sexual reduce el riesgo de contraer el VPH y otras infecciones de transmisión sexual (ITS).

- Limitar el número de compañeros sexuales que tenga y evitar las parejas que participen en actividades sexuales de alto riesgo.

- Hacerse citologías vaginales con la frecuencia que 
el médico le recomiende. Las citologías vaginales pueden ayudar a detectar cambios precoces, los cuales pueden tratarse antes de que se conviertan en cáncer cervical. Es importante practicarse esta prueba para tener la seguridad de que no existe ningún problema de cáncer en el cuello de la matriz, la prueba del Papanicolaou ayuda a detectar cualquier anormalidad y así estar a tiempo de seguir un tratamiento en caso de existir algún riesgo de cualquier enfermedad.

El problema que dio origen a esta investigación ha estado relacionado a la falta de asistencia directa a un grupo poblacional con información sobre prevención del cáncer de Cérvix, para lo cual se acudió al puesto tenerlo como una edad avanzada (mayores de 45 años).

Entre los problemas identificados se tienen, los aspectos culturales como la falta de conocimiento y desinterés de la población sobre la forma de prevención del virus de cáncer de cuello uterino además de las pruebas que se realizan como el test de Papanicolaou y falta de campañas informativas para la toma del Papanicolaov; también la falta de personal capacitado para la realización masiva de Papanicolaou y el desinterés del personal sobre la población de Incho respecto a la aplicación masiva del Papanicolaou; también sobre los materiales y equipamiento del puesto de salud de Incho se tomó conocimiento sobre la falta

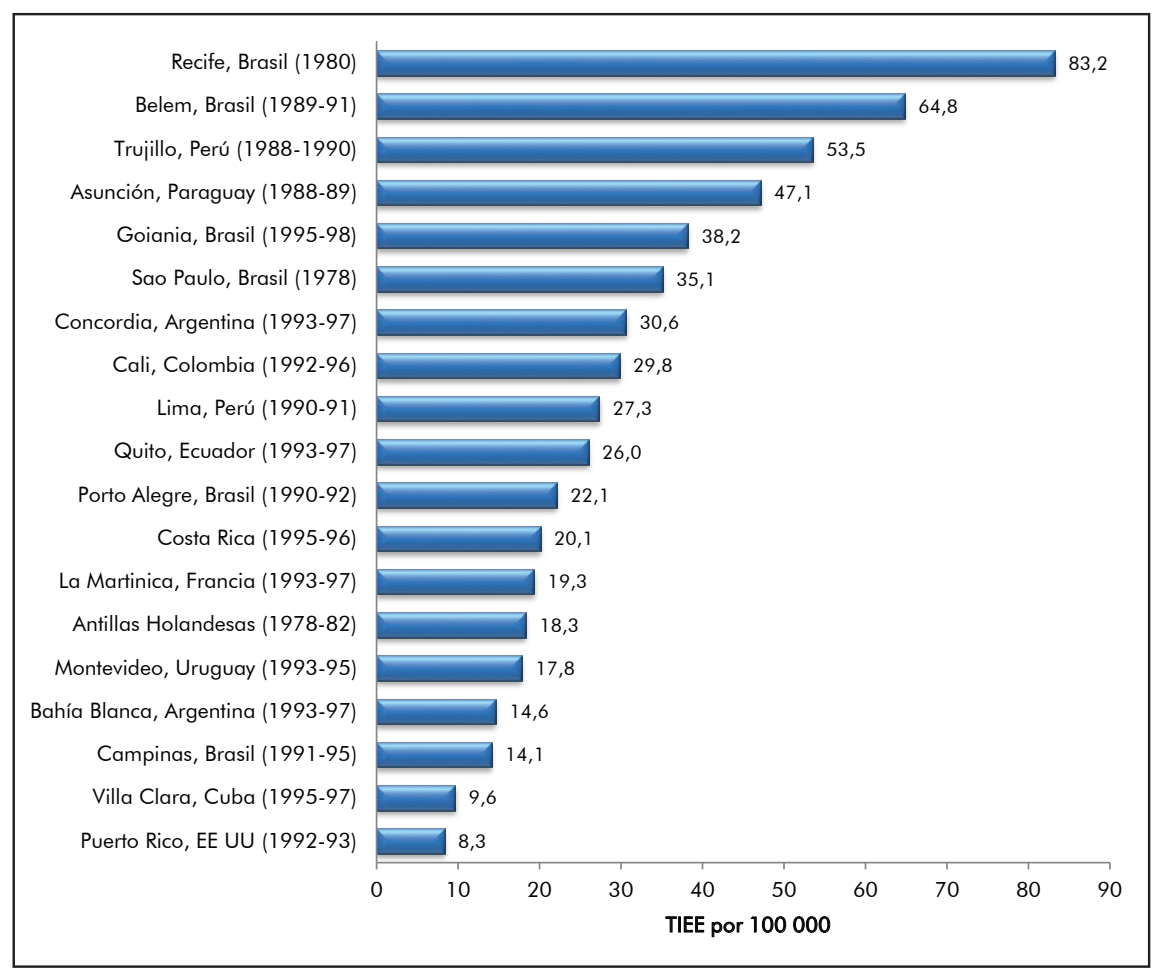

Figura $\mathrm{N}^{\circ}$ 2: Incidencia de cáncer de cérvix en registros de cáncer de América Latina

Fuente: http://sisbib.unmsm.edu.pe/bvrevistas/ginecologia/vol53_n2/pdf/A05V53N2.pdf

de salud de Incho, aplicando el ASIS (Análisis de situación de salud), para identificación de problemas que afecta la calidad de salud de la población, diagnosticando los problemas demográficos, determinantes ambientales, determinantes económicos, y determinantes asociados a la oferta y la demanda de la población con el puesto de salud. Se identificó que la cantidad de exámenes realizados en el puesto de salud de Incho ha sido relativamente baja, siendo muy importante estos exámenes para la prevención de procesos cancerígenos u oncológicos, que pueden ser prevenibles con la realización de el "Test de Papanicolaou", si es realizado a mujeres que ya han tenido o mantienen una vida sexual activa, y están dentro de una población muy propensas a de organización y disposición de materiales para la realización masiva de Papanicolaou; finalmente el escaso presupuesto para la compra de insumos.

Se identificó que la cantidad de exámenes realizados en el puesto de salud de Incho es baja, siendo importante estos exámenes para la prevención de procesos cancerígenos $u$ oncológicos, que pueden ser prevenibles con la realización del el test Papanicolaou si se realiza a mujeres que ya han tenido o mantienen una vida sexual activa. 


\section{Campaña preventiva de cáncer de Cérvix}

La población en la cual se intervino estuvo compuesta por madres de familia del anexo de Incho en el distrito de El Tambo, Huancayo. Se empleó material impreso (afiches y volantes) para tener la participación voluntaria de las madres en Incho, el local de la reunión fue el Colegio "Corazón de Jesús", ubicado en el Psje. Agusto Salazar Bondy N 250, Urb. Siglo XX. Se logró tener información de 70 madres de familia.

Para la recolección de datos se utilizó un cuestionario semi estructurado que constó de 10 preguntas las cuales incluyeron: datos generales de la pobladora asistente (Nombre y edad), y preguntas sobre si antes de la charla recibida conocía algunos conceptos de prevención, los test que se pueda realizar, y no había recibido anteriormente charlas informativas de cómo prevenir el Cáncer de Cuello Uterino, ni sabían sus formas de prevención. Pero sin embargo la mayoría de esta población sabía que el test de Papanicolaou se realiza para hacer despistajes de cáncer de cérvix. Desconocían cada cuanto tiempo se tenían que realizar este Test.

De la población encuestada la mayoría no se había realizado en ninguna oportunidad el test de Papanicolaou.

Absolutamente toda la población después de haber recibido la charla quedó con el compromiso de asistir al centro de salud más cercano para realizarse el test de Papanicolaou.

- Con los resultados en las encuestas, la mayor

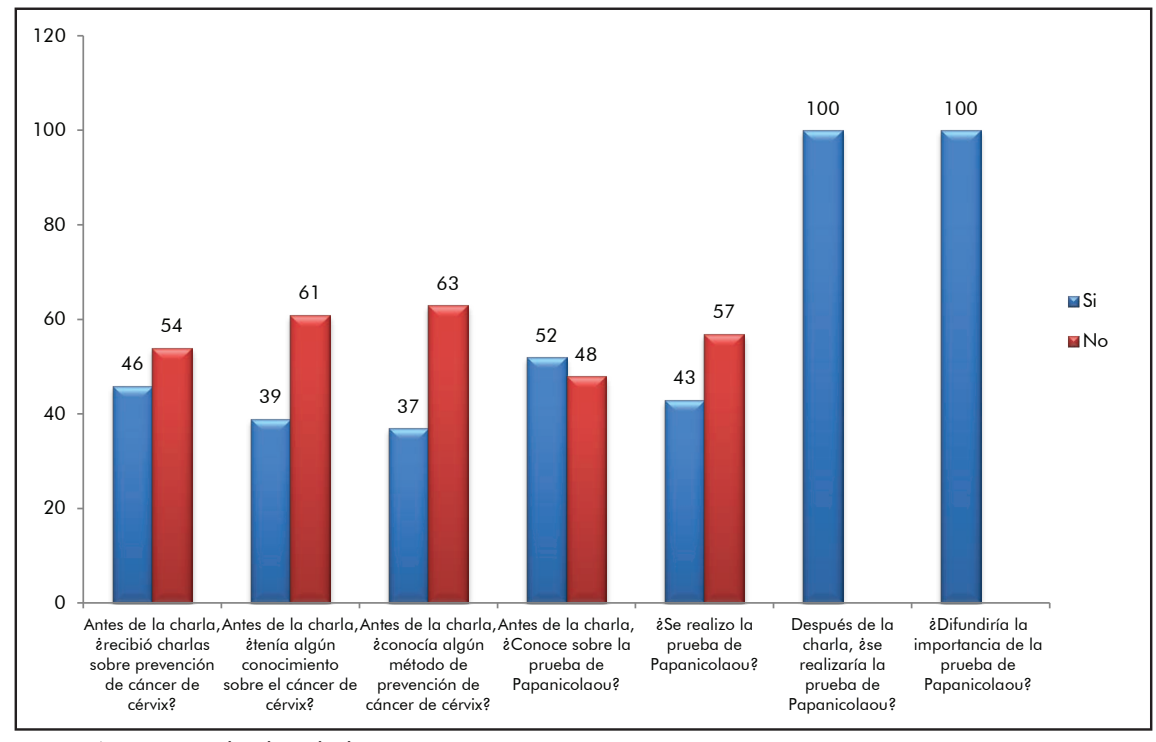

Figura $\mathrm{N}^{\circ}$ 3: Resultados de la encuesta

cada cuanto tiempo debería realizarse, además de preguntas abiertas sobre la opinión de estos eventos. Asimismo se empleó la espina de Ishikawa y la tabla de priorización de problemas frente a las soluciones que se estaban planteando, (figura 3).

El cuestionario fue aplicado a todas las madres, jóvenes y púberes asistentes, luego se les entregó un globo y unja cinta (significado de la lucha contra el cáncer de Cuello Uterino). Dentro de este evento se empleó juegos, y gigantografías para que relacionen de manera general lo que una mujer debe saber para prevenir el cáncer de cérvix (prevención, promoción, signos de alarma, y exámenes auxiliares)

\section{Algunos hallazgos importantes}

La mayoría de las madres asistentes a esta campaña parte de la población no había recibido una charla informativa que abarcara a bastantes madres.

- La mayoría de las madres lo que llamo la atención fue que las mujeres asistentes a la campaña la gran mayoría no sabían manera de cómo prevenir en cáncer de cuello uterino.

- La mayoría de madres no sabían o no conocían de factores de riesgo que predisponen el cáncer de cuello uterino.

- La mayoría de madres si sabían sobre la realización de la prueba de Papanicolaou.

- Más de la mitad de las madres asistentes nos manifiestan que alguna vez se realizaron la prueba de Papanicolaou. 
- Todas las madres que realizaron la encuesta coincidieron en realizarse la prueba de Papanicolaou

- Conjuntamente las madres concuerdan con difundir a sus conocidas sobre cómo prevenir el cáncer de cuello uterino.

Se entenderá que las mujeres que se realizarán estos despistajes, muchas de ellas tendrán lesiones precancerosas, y ya no desarrollaran la enfermedad, porque se tomaran medidas respectivas frente a esas lesiones precancerosas. $Y$ eso disminuye los costos a nuestra región como el tratamiento de una quimioterapia que sale mucho más costoso que una crioterapia o una histerectomía.

\section{CONCLUSIONES}

Este tipo de campañas ayuda a sensibilizar y a difundir a poblaciones expensas a contraer enfermedades, no solo el cáncer de cuello uterino.

Frente a esta campaña se logró identificar población vulnerable que se logró aumentar las demandas de despistaje de Cáncer de Cuello Uterino.

Esta población se ha sentido satisfecha de haber recibido esta clase de información, que para ellos es de difícil acceso. Incentivando a este tipo de proyectos se lograra a disminuir una cantidad muy considerable sobre la incidencia de cáncer de cérvix, así como otras enfermedades prevenibles.

Siendo un gran problema de salud pública, prevenible en la gran mayoría de casos, los volantes y boletines repartidos nos ayudan a concretar mucha información. Así los pobladores (madres, jóvenes y púberes) se pueden llevar a sus casas estos boletines informativos para que puedan volverlo a leer si alguna información no les ha quedado claro.
Todas las madres que realizaron la encuesta coincidieron en realizarse la prueba de Papanicolaou de acuerdo a nuestra encuesta empleada.

Conjuntamente las madres concuerdan con difundir a sus conocidas sobre cómo prevenir el cáncer de cuello uterino como manera de seguir previniendo y promocionando a las personas a las que no se pudo llegar.

\section{REFERENCIAS BIBLIOGRÁFICAS}

1. Matos E, Loria D, et al. Prevalence of Human Papillomarivus Infection Among Women in Concordia, Argentina. Sexually Transmitted Diseases. 2003; 30(8): 593-599.

2. Sharpe $P$, Brandt H, McCree D. Knowledge and beliefs about abnormal pap test results and HPV among women with highrisk HPV: results from indepth interviews. Women Health. 2005; 42(2): 107-133.

3. Bosch FX, Lorincz A, Munoz N, Meijer CJ, Shah KV. The causal relation between human papillomavirus and cervical cancer. J Clin Pathol. 2002; 55(4): 244-265.

4. Ministerio de Salud de la Nación. Recomendaciones para el tamizaje, seguimiento y tratamiento de mujeres para la prevención del cáncer cervicouterino en el marco de la incorporación de la prueba de VPH como tamizaje primario en Argentina. Buenos Aires: Instituto Nacional del cáncer; 2011.

5. Rivera R, Aguilera J, Larraín A. Epidemiologia del Virus Papiloma Humano (HPV). Rev Chil Obstet Ginecol. 2002; 67(6): 501-506.

6. Salmerón J, Lazcano-Ponce $E$, Lorincz A, Hernández M, Hernández $P$, et al. Comparison of HPV-based assays with Papanicolaou smears for cervical cancer screening in Morelos State, México. Cancer Causes Control, 2003; 14, 505-551. 